\title{
Modelling the Impact of Media in Controlling the Diseases with a Piecewise Transmission Rate
}

\author{
Maoxing Liu, Yuting Chang, and Lixia Zuo \\ Department of Mathematics, North University of China, Taiyuan, Shanxi 030051, China \\ Correspondence should be addressed to Maoxing Liu; liumxsx@gmail.com
}

Received 23 October 2015; Accepted 5 January 2016

Academic Editor: Amit Chakraborty

Copyright (C) 2016 Maoxing Liu et al. This is an open access article distributed under the Creative Commons Attribution License, which permits unrestricted use, distribution, and reproduction in any medium, provided the original work is properly cited.

An epidemic model with media is proposed to describe the spread of infectious diseases in a given region. A piecewise continuous transmission rate is introduced to describe that the media has its effect when the number of the infected exceeds a certain critical level. Furthermore, it is assumed that the impact of the media on the contact transmission is described by an exponential function. Stability analysis of the model shows that the disease-free equilibrium is globally asymptotically stable if the basic reproduction number is less than unity. On the other hand, when the basic reproduction number is greater than unity, a unique endemic equilibrium exists, which is also globally asymptotically stable. Our analysis implies that media coverage plays an important role in controlling the spread of the disease.

\section{Introduction}

When an infectious disease outbreaks, the spread and the control of the disease will be reported by the media including television programs, newspapers, and online social networks. Many examples are the massive reports and daily updates in the public media on the number of the infections and deaths, which had important impacts on the diseases control $[1,2]$. It is shown that media coverage plays an important role in the spread and control of the infectious disease, such as the SARS in 2003 [3], the H1N1 in 2009 influenza epidemic [4, 5], and the Ebola in 2014 in Africa [6].

Recently, such impact on disease spreading and controlling has been investigated by mathematical modeling approach [5, 7-20]. In these research works some focused their attention on the incidence rate, and the recent survey identified three typical terms in [5]. Liu et al. [7] described the impact of media coverage using the transmission coefficient $\beta e^{-\alpha_{1} E-\alpha_{2} I-\alpha_{3} H}$, and this impact leads to the change of avoidance and contact patterns at both individual and community levels. Cui et al. [8-11] developed a compartment model using incidence rate $\mu e^{-m I} S I$ with $m>0$ to investigate the impact of media coverage on the transmission, and stability analysis of the models has shown that Hopf bifurcation can occur. Tchuenche et al. [14] used an exponentially decreasing function $e^{-M(t)}$ to reveal the force of media and showed the potential short-term beneficial effect of awareness programs.

More recently nonsmooth media functions [21-25] have been studied. Xiao et al. [21] introduced a segmented function to describe the media impact $e^{-m I_{c}}$, here $m$ is also the strength of the media effect, and $I_{c}$ is a threshold that people take the controlling measures or not. A Filippov epidemic model was proposed to describe the real characteristics of media impact in the spread of infectious diseases by incorporating a piecewise continuous transmission rate $\beta e^{(-\alpha \epsilon I)} S I$ in [24], and mathematical analysis with regard to the local and global stability of equilibria and local sliding bifurcations are performed.

In fact, during infectious disease outbreaks, individuals may reduce their activities after receiving information about the risk of infection. For example, people will reduce the time that they go out, students will not attend school, and so on, and such information on the ongoing epidemics may impact the dynamics itself. In fact, at the initial stages of the prevalence of disease, most people and public mass media are unaware of the disease; thus, the individuals will not do any protective measures. Only when the number of infectious individuals reaches and exceeds a certain level, the individuals will take precautionary measures against 
the diseases. Based on the above facts, in this paper we also focus on the incidence rate. Here we introduce $\sigma(I)=$ $I-I_{c}$ to show this fact, where $I_{c}$ is the threshold value. It is assumed that the impact of media is described by an exponential decreasing factor and the population obeys the logistic growth.

The rest of this paper is organized as follows: in the next section, a mathematical model is proposed in order to reveal the effect of media; then the existence of equilibria is given in Section 3. In Section 4 the local and global stability of the disease-free and the unique endemic equilibria are analyzed. Furthermore, in Section 5 some numerical simulations and discussions are given in the last section.

\section{Mathematical Model with Media}

In this model the population is divided into three types: the susceptible, the infective and the recovered. Let $S(t), I(t)$, and $R(t)$ denote the number of susceptible, infective, and recovered individuals at time $t$, respectively. It is assumed that the growth of the susceptible population obeys the logistic growth, the intrinsic growth rate of the population is $b$, and the carrying capacity for the population is $K$. The interactions between susceptible and infective individuals are assumed to be bilinear, and $\beta$ is the contact rate of susceptible with infective individuals, $\gamma$ is the recovery rate of the infective individuals, and $d$ is the natural death rate of the population. Consider that the cumulative density of media about the disease is $M(t)$. The growth rate of cumulative density of media, $\sigma$, is assumed to be proportional to the number of infective individuals in the population, and $v$ is the depletion rate of cumulative density of media. Thus, we have the following model:

$$
\begin{aligned}
S^{\prime}(t) & =b S\left(1-\frac{S}{K}\right)-\beta S I, \\
I^{\prime}(t) & =\beta S I-(\gamma+d) I, \\
R^{\prime}(t) & =\gamma I-d R, \\
M^{\prime}(t) & =\sigma I-\nu M .
\end{aligned}
$$

In this model we assume that the impact of media is described by an exponential decreasing factor as $\beta=$ $\beta_{0} e^{-\epsilon m M}$, where the parameter $m>0$ is to reflect the impact of media. When $\epsilon=0$, the transmission rate is the constant $\beta_{0}$. When $\epsilon=1$, as one can see if $m$ is relatively small, the transmission rate $\beta$ is close to the constant $\beta_{0}$. Thus, the model can be rewritten as follows:

$$
\begin{aligned}
S^{\prime}(t) & =b S\left(1-\frac{S}{K}\right)-\beta_{0} e^{-\epsilon m M} S I, \\
I^{\prime}(t) & =\beta_{0} e^{-\epsilon m M} S I-(\gamma+d) I, \\
R^{\prime}(t) & =\gamma I-d R \\
M^{\prime}(t) & =\sigma I-v M
\end{aligned}
$$

with

$$
\epsilon= \begin{cases}0, & \sigma(I) \leq 0 \\ 1, & \sigma(I)>0\end{cases}
$$

where the function $\sigma(I)=I-I_{c}$ means that when infective individuals reach and exceed the certain level $I_{c}$, the media has its effects.

For the solutions of system (2) with (3), the region of attraction is given by the set:

$$
\begin{aligned}
\Omega & =\left\{(S, I, R, M) \in R_{+}^{4} \mid 0<S(t)+I(t)+R(t)\right. \\
& \left.\leq K, 0 \leq M \leq \frac{\sigma K}{v}\right\},
\end{aligned}
$$

and it attracts all solutions initiating in the interior of the positive orthant. The $(S, I, R, M)$ phase space is split into two parts: $G_{1}=\left\{(S, I, R, M) \in R_{+}^{4} \mid \sigma(I) \leq 0\right\}$ and $G_{2}=$ $\left\{(S, I, R, M) \in R_{+}^{4} \mid \sigma(I)>0\right\}$. In region $G_{1}$, there is no effect of the media and the transmission rate $\beta=\beta_{0}$, while in region $G_{2}$, the transmission rate declines to $\beta_{0} e^{-m M}$.

For convenience, let the vector $Z=(S, I, M)^{T}$, and denote

$$
\begin{aligned}
& F_{G_{1}}(Z)=\left(b S\left(1-\frac{S}{K}\right)-\beta_{0} S I, \beta_{0} S I-(\gamma+d) I, \sigma I\right. \\
& -v M)^{T}, \\
& F_{G_{2}}(Z)=\left(b S\left(1-\frac{S}{K}\right)-\beta_{0} e^{-m M} S I, \beta_{0} e^{-m M} S I\right. \\
& -(\gamma+d) I, \sigma I-\nu M)^{T} .
\end{aligned}
$$

Then system (2) with (3) can be rewritten as the following switching system:

$$
Z^{\prime}= \begin{cases}F_{G_{1}}(Z), & Z \in G_{1}, \\ F_{G_{2}}(Z), & Z \in G_{2} .\end{cases}
$$

In the following, system (6) defined in region $G_{1}$ is the subsystem $S_{1}$ and that defined in region $G_{2}$ is the subsystem $S_{2}$, and we consider the two subsystems in isolation firstly.

\section{Existence of Equilibria}

3.1. Equilibria of $S_{1}$. Let $F_{G_{1}}(Z)$ be zero; one can verify that the origin $E_{0}^{1}=(0,0,0)$ is a hyperbolic saddle point with eigenvalues $b,-(\gamma+d),-\nu$. The subsystem $S_{1}$ has one diseasefree equilibrium $E_{0}^{2}=(K, 0,0)$, and the stability of $E_{0}^{2}$ can be obtained in the following section. 
It follows from [26] that the reproduction number $R_{0}=$ $\beta_{0} K /(\gamma+d)$ and we can verify that when $R_{0}>1, S_{1}$ has a unique endemic equilibrium $E_{0}^{*}=\left(S_{0}^{*}, I_{0}^{*}, M_{0}^{*}\right)$, where

$$
\begin{aligned}
S_{0}^{*} & =\frac{\gamma+d}{\beta_{0}}, \\
I_{0}^{*} & =\frac{b}{\beta_{0}}\left(1-\frac{1}{R_{0}}\right), \\
M_{0}^{*} & =\frac{\sigma}{v} I_{0}^{*} .
\end{aligned}
$$

3.2. Equilibria of $S_{2}$. The disease-free equilibria of the subsystem $S_{2}$ are same with the subsystem $S_{1}$; thus, we study the existence of the endemic equilibrium of $S_{2}$.

Let $F_{G_{2}}(Z)$ be zero; one can see that the endemic equilibrium must satisfy

$$
\begin{aligned}
b S\left(1-\frac{S}{K}\right)-\beta_{0} e^{-m M} S I & =0, \\
\beta_{0} e^{-m M} S & =\gamma+d, \\
\sigma I-\nu M & =0 .
\end{aligned}
$$

If there exists a positive equilibrium, it is a positive solution of

$$
\begin{aligned}
& S=K\left(1-\frac{\beta_{0}}{b} I e^{-(m \sigma / v) I}\right):=g(I), \\
& S=\frac{K}{R_{0}} e^{(m \sigma / v) I}:=h(I) .
\end{aligned}
$$

One can verify that $g(0)=K, h(0)=K / R_{0}$, and if $R_{0}>1$, $g(0)>h(0)$. Hence, the two curves $S=g(I)$ and $S=h(I)$ have at least one positive intersection. In order to determine the number of positive intersections we consider the tangency of two curves. If the two curves intersect, it must have $g(I)=$ $h(I), g^{\prime}(I)=h^{\prime}(I)$; that is,

$$
\begin{aligned}
1-\frac{\beta_{0}}{b} I e^{-(m \sigma / v) I} & =\frac{1}{R_{0}} e^{(m \sigma / v) I}, \\
\frac{\beta_{0}}{b} e^{-(m \sigma / v) I}\left(\frac{m \sigma}{v} I-1\right) & =\frac{m \sigma}{R_{0} v} e^{(m \sigma / v) I} .
\end{aligned}
$$

From (10) I must satisfy the quadratic equation:

$$
\left(2 I-\frac{v}{m \sigma}\right)^{2}=\frac{b R_{0}}{\beta_{0}}\left(I-\frac{v}{m \sigma}\right) .
$$

Let $\delta=b R_{0} / \beta_{0}, \alpha=\nu / m \sigma$; then we have

$$
I=\frac{4 \alpha+\delta \pm \sqrt{\delta(\delta-8 \alpha)}}{8}
$$

Let $m_{0}=8 \beta_{0} v / b \sigma R_{0}$; if $0<m<m_{0}$, (11) has no roots and the system has a unique endemic equilibrium. If $m=m_{0}$, (11) has one unique root and the system has one endemic equilibria of multiplicity at least two. If $m>m_{0}$, (11) has two roots and the system has three endemic equilibria. Thus, we have the following theorem.
Theorem 1. When $R_{0}>1$ and $0<m<m_{0}$ subsystem $S_{2}$ has a unique endemic equilibrium $E_{*}=\left(S^{*}, I^{*}, M^{*}\right)$.

\section{Stability of Equilibria}

In this section we present the locally and globally asymptotical stability of the equilibria of subsystem $S_{1}$ and subsystem $\mathrm{S}_{2}$.

Theorem 2. The disease-free equilibrium $E_{0}^{2}$ is locally asymptotically stable if $R_{0}<1$ and unstable when $R_{0}>1$.

Proof. The characteristic equation corresponding to the subsystem $S_{1}$ at $E_{0}^{2}$ is the form

$$
(\lambda+b)\left[\lambda-\beta_{0} K+(\gamma+d)\right](\lambda+\nu)=0,
$$

where $\lambda$ is the eigenvalue. We get

$$
\begin{aligned}
& \lambda_{1}=-b, \\
& \lambda_{2}=\beta_{0} K-(\gamma+d)=(\gamma+d)\left(R_{0}-1\right), \\
& \lambda_{3}=-\nu .
\end{aligned}
$$

Thus, $E_{0}^{2}$ is locally asymptotically stable if $R_{0}<1$ and unstable when $R_{0}>1$. The proof of the disease-free equilibrium of the subsystem $S_{2}$ is similar to $S_{1}$; then this proof is omitted.

Theorem 3. The disease-free equilibrium $E_{0}^{2}$ is globally asymptotically stable if $R_{0}<1$

Proof. To establish the global stability of the disease-free equilibrium of subsystem $S_{2}$, we consider the positive definite function $V(t)=I(t)$ by using Lyapunov's method. Now differentiating $V$ with respect to $t$, we get

$$
\begin{aligned}
\frac{d V}{d t} & =\left[\beta e^{-m M} S-(\gamma+d)\right] I<[\beta S-(\gamma+d)] I \\
& =\left(R_{0}-1\right)(\gamma+d) I .
\end{aligned}
$$

If $R_{0}<1$, then $d V / d t \leq 0$. Thus, LaSalle's invariance principle implies that $E_{0}^{2}$ is globally asymptotically stable in $\Omega$. We can use the same positive definite function when we prove the global stability of the disease-free equilibrium of subsystem $S_{1}$.

In fact, when $R_{0}<1$ curves starting from $G_{2}$ will converge to $(K, 0,0)$ that belongs to $G_{1}$, and curves starting from $G_{1}$ also converge to $(K, 0,0)$; therefore, $E_{0}^{2}=(K, 0,0)$ is globally asymptotically stable. In the following we consider the stability of the endemic equilibrium $E_{0}^{*}$ of subsystem $S_{1}$ and $E_{*}$ of subsystem $S_{2}$. According to Hurwitz criterion, it is easy to get the following proposition.

Proposition 4. The unique endemic equilibrium $E_{0}^{*}$ of subsystem $S_{1}$ is locally asymptotically stable if $R_{0}>1$. The unique endemic equilibrium $E_{*}$ of subsystem $S_{2}$ is locally asymptotically stable if $R_{0}>1$. 


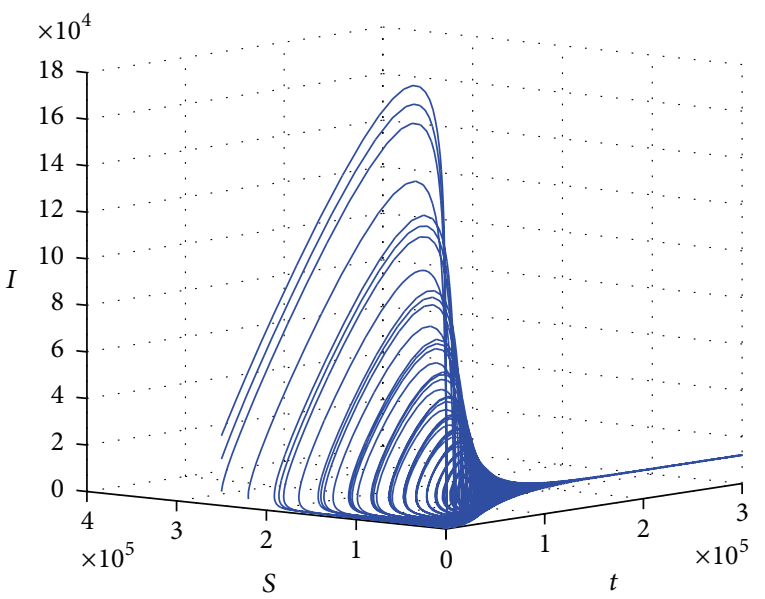

(a) Global stability of $E_{0}^{*}$ when $1<R_{0}<R_{c}$

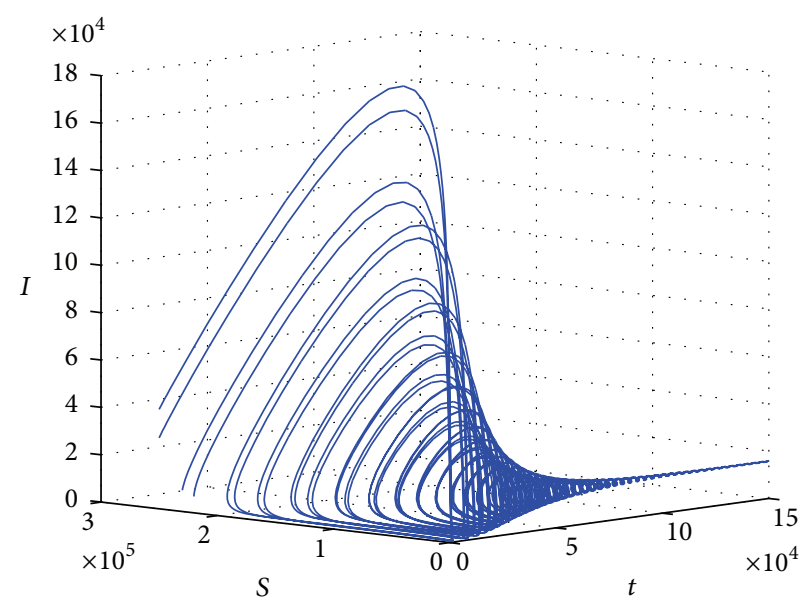

(b) Global stability of $E_{*}$ when $R_{0}>R_{c}$

FIgURE 1: The stability of the endemic equilibria $E_{0}^{*}$ and $E_{*}$.

When the endemic equilibrium $E_{0}^{*}$ belongs to $G_{1}$, we get $1<R_{0}<b /\left(b-\beta_{0} I_{c}\right)$ from the expression of $I_{0}^{*}$, so if $R_{0}>$ $b /\left(b-\beta_{0} I_{c}\right), E_{0}^{*}$ belongs to $G_{2}$, which lead to the existence of $E_{*}$ that belongs to $G_{2}$. Let $R_{c}=b /\left(b-\beta_{0} I_{c}\right)$; then we have the following result.

Theorem 5. When $1<R_{0}<R_{c}$ the endemic equilibrium $E_{0}^{*}$ is globally asymptotically stable; when $R_{0}>R_{c}$ the endemic equilibrium $E_{*}$ is globally asymptotically stable.

Proof. Let a Dulac function

$$
D(S, I, M)=\frac{1}{S I},
$$

and we make $f_{1}=b(1-S / K) S-\beta_{0} e^{-m M} S I, f_{2}=\beta_{0} e^{-m M} S I-$ $(\gamma+d) I$, and $f_{3}=\sigma I-v M$ for the subsystem $S_{2}$. Then

$$
\frac{\partial\left(D f_{1}\right)}{\partial S}+\frac{\partial\left(D f_{2}\right)}{\partial I}+\frac{\partial\left(D f_{3}\right)}{\partial M}=-\frac{b}{K I}-\frac{\nu}{S I}<0 .
$$

Hence, we can exclude the existence of limit cycles in region $G_{2}$. The same method can be applied in discussion for region $G_{1}$.

When $1<R_{0}<R_{c}$, it is easy to get the endemic equilibrium $E_{0}^{*}$ that belongs to $G_{1}$ and all curves starting from $G_{1}$ will verge to $E_{0}^{*}$. We know that all curves starting from $G_{2}$ will converge to $E_{*}$, but $E_{*}$ also belongs to $G_{1}$ if $1<R_{0}<R_{c}$, and there is a unique endemic equilibrium and no limit cycles in region $G_{1}$; hence, curves starting from $G_{2}$ will enter $G_{1}$ and converge to $E_{0}^{*}$. Thus, when $1<R_{0}<$ $R_{c}$ the endemic equilibrium $E_{0}^{*}$ is globally asymptotically stable. Similarly when $R_{0}>R_{c}$ the endemic equilibrium $E_{0}^{*}$ belongs to $G_{2}$; hence, curves starting from $G_{1}$ will enter $G_{2}$ and converge to the unique endemic equilibrium $E_{*}$ in region $G_{2}$. So when $R_{0}>R_{c}$ the endemic equilibrium $E_{*}$ is globally asymptotically stable.

\section{Numerical Simulations and Results}

To check the analysis in Section 4 about $E_{0}^{*}$ and $E_{*}$, we do some numerical simulations in this section.

When $1<R_{0}<R_{c}$, we set the following values of parameters: $b=0.00095, K=500000, \beta_{0}=0.0000001, m=0.05, \gamma=$ $0.003, d=0.0002$, and $I_{c}=9000$, and we get $R_{0}=8.8, R_{c}=$ 12.3 , and $1<R_{0}<R_{c}$. Next we consider another case $R_{0}>R_{c}$ by taking $b=0.001, \beta_{0}=0.00000009$, and $I_{c}=10000$, and get $R_{0}=13.2, R_{c}=10$. We, respectively, choose two sets of initial values that belong to $G_{1}\left(I_{0}<I_{c}\right)$ and two sets of initial values that belong to $G_{2}\left(I_{0}>I_{c}\right)$. Thus, we get $I_{0}^{*}=8771<9000$, $I_{*}=10680>10000$; see Figure 1. As shown in Figure 1(a) the trajectories all approach towards $E_{0}^{*}$ no matter whether $I_{0} \epsilon$ $G_{1}$ or $G_{2}$. Hence, $E_{0}^{*}$ is globally asymptotically stable when $1<$ $R_{0}<R_{c}$. Whereas when $R_{0}>R_{c}$ the trajectories approach towards $E_{*}$ what Figure $1(\mathrm{~b})$ responses. In conclusion, $E_{0}^{*}$ and $E_{*}$ are globally asymptotically stable if $R_{0}>1$.

In the following we consider the impact of media coverage on the spread of diseases by drawing the curves between $I(t), S(t)$, and $m$, respectively, as shown in Figure 2. It is shown that the number of $I(t)$ reduces with the increase of $m$, which is opposite to $S(t)$. This is common sense that media coverage influences people's behavior and reminds them to prevent themselves from the diseases through staying at home, reducing travel or quarantine, and so on. So it is clear that media coverage plays a key role in the preventing and controlling the diseases.

\section{Discussion}

In this paper, a nonlinear mathematical model has been proposed and analyzed to describe the impact of media coverage on the transmission dynamics of infectious diseases. Considering the fact that public mass media usually do not work when the number of the infected individuals is generally small at the initial stages of prevalence of the disease, and by incorporating a piecewise continuous transmission rate to represent that media coverage has its effects only when 


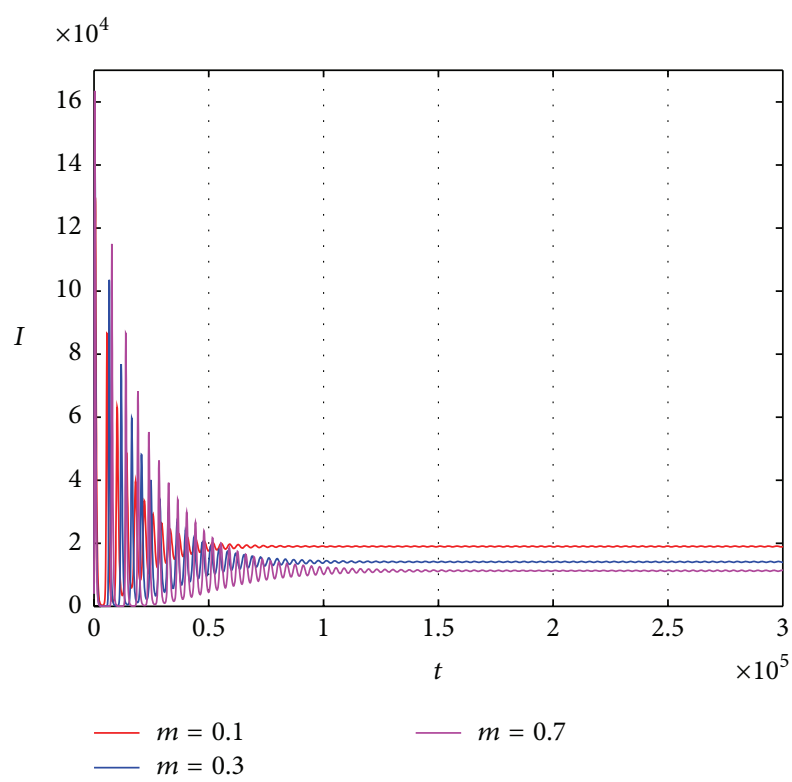

(a) Number of the infected individuals

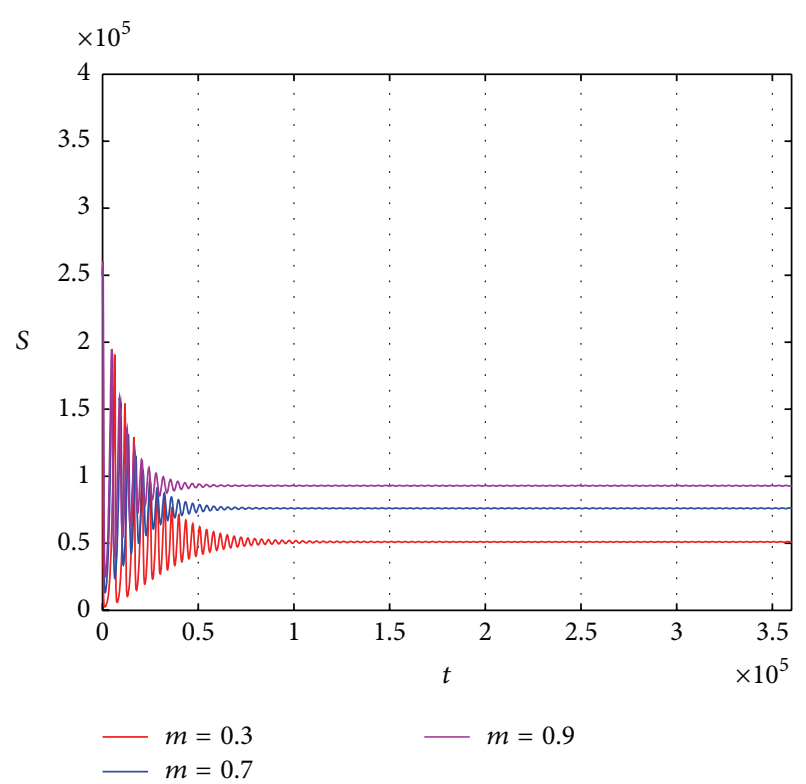

(b) Number of the susceptible individuals

FIgURE 2: The curves of $I(t)$ and $S(t)$ with different $m$.

the number of the infected individuals exceeds a certain level. The disease-free equilibrium has been shown to be globally asymptotically stable when the basic reproduction number $R_{0}<1$. When $R_{0}>1$ and $0<m<m_{0}$ sufficiently small, it leads to the global stability of endemic equilibrium. But when $1<R_{0}<R_{c}$ the trajectories all approach towards $E_{0}^{*}$ whenever initial starts $\in G_{1}$ or $G_{2}$, and the trajectories approach towards $E_{*}$ if $R_{0}>R_{c}$. Numerical simulations prove the stability of equilibria and the impact of media coverage in the spread of diseases, which suggest that if we want to reduce the number of the infected individuals, we should increase media coverage.

\section{Conflict of Interests}

The authors declare that there is no conflict of interests regarding the publication of this paper.

\section{Acknowledgments}

The project is supported by the National Sciences Foundation of China (11571324) and the Scientific Activities of Selected Returned Overseas Professionals in Shanxi Province.

\section{References}

[1] M. Brodie, E. Hamel, L. A. Brady, J. Kates, and D. E. Altman, AIDS at 21: Media Coverage of the HIV Epidemic 1981-2002, Princeton Survey Research Associates International, 2004.

[2] J. T. F. Lau, M. Lau, J. H. Kim, and H. Y. Tsui, "Impacts of media coverage on the community stress level in Hong Kong after the tsunami on 26 December 2004," Journal of Epidemiology and Community Health, vol. 60, no. 8, pp. 675-682, 2006.
[3] A. B. Gumel, S. Ruan, T. Day et al., "Modelling strategies for controlling SARS outbreaks," Proceedings of the Royal Society B: Biological Sciences, vol. 271, no. 1554, pp. 2223-2232, 2004.

[4] C. Fraser, C. A. Donnelly, S. Cauchemez et al., "Pandemic potential of a strain of influenza A (H1N1): early findings," Science, vol. 324, no. 5934, pp. 1557-1561, 2009.

[5] S. Collinson and J. M. Heffernan, "Modelling the effects of media during an influenza epidemic," BMC Public Health, vol. 14, pp. 376-386, 2014.

[6] M. S. Majumder, S. Kluberg, M. Santillana, S. Mekaru, and J. S. Brownstein, "Ebola outbreak: media events track changes in observed reproductive number," PLoS Currents Outbreaks, 2015.

[7] R. S. Liu, J. H. Wu, and H. P. Zhu, "Media/psychological impact on multiple outbreaks of emerging infectious diseases," Computational and Mathematical Methods in Medicine, vol. 8, no. 3, pp. 153-164, 2007.

[8] J. A. Cui, Y. H. Sun, and H. P. Zhu, "The impact of media on the control of infectious diseases," Journal of Dynamics and Differential Equations, vol. 20, no. 1, pp. 31-53, 2008.

[9] Y. Liu, J. A. Cui, and H. Zhu, "The impact of media coverage on the dynamics of infectious disease," International Journal of Biomathematics, vol. 1, no. 1, pp. 65-74, 2008.

[10] J.-A. Cui, X. Tao, and H. P. Zhu, "An SIS infection model incorporating media coverage," The Rocky Mountain Journal of Mathematics, vol. 38, no. 5, pp. 1323-1334, 2008.

[11] Y. Li and J. Cui, "The effect of constant and pulse vaccination on SIS epidemic models incorporating media coverage," Communications in Nonlinear Science and Numerical Simulation, vol. 14, no. 5, pp. 2353-2365, 2009.

[12] S. Funk, E. Gilad, C. Watkins, and V. A. A. Jansen, "The spread of awareness and its impact on epidemic outbreaks," Proceedings of the National Academy of Sciences of the United States of America, vol. 106, no. 16, pp. 6872-6877, 2009.

[13] W. Liu, "A SIRS epidemic model incorporating media coverage with random perturbation," Abstract and Applied Analysis, vol. 2013, Article ID 792308, 9 pages, 2013. 
[14] J. M. Tchuenche, N. Dube, C. P. Bhunu, R. J. Smith, and C. T. Bauch, "The impact of media coverage on the transmission dynamics of human influenza," BMC Public Health, vol. 11, article S5, 2011.

[15] S. Samanta, S. Rana, A. Sharma, A. K. Misra, and J. Chattopadhyay, "Effect of awareness programs by media on the epidemic outbreaks: a mathematical model," Applied Mathematics and Computation, vol. 219, no. 12, pp. 6965-6977, 2013.

[16] F. Nyabadza, C. Chiyaka, Z. Mukandavire, and S. D. HoveMusekwa, "Analysis of an HIV/AIDS model with public-health information campaigns and individual withdrawal," Journal of Biological Systems, vol. 18, no. 2, pp. 357-375, 2010.

[17] A. K. Misra, A. Sharma, and J. B. Shukla, "Modeling and analysis of effects of awareness programs by media on the spread of infectious diseases," Mathematical and Computer Modelling, vol. 53, no. 5-6, pp. 1221-1228, 2011.

[18] Y. Wang, J. Cao, Z. Jin, H. Zhang, and G.-Q. Sun, "Impact of media coverage on epidemic spreading in complex networks," Physica A: Statistical Mechanics and its Applications, vol. 392, no. 23, pp. 5824-5835, 2013.

[19] X. Yuan, Y. Xue, and M. Liu, "Analysis of an epidemic model with awareness programs by media on complex networks," Chaos, Solitons \& Fractals, vol. 48, no. 1, pp. 1-11, 2013.

[20] G.-Q. Sun, S.-L. Wang, Q. Ren, Z. Jin, and Y.-P. Wu, "Effects of time delay and space on herbivore dynamics: linking inducible defenses of plants to herbivore outbreak," Scientific Reports, vol. 5, Article ID 11246, 2015.

[21] Y. Xiao, X. Xu, and S. Tang, "Sliding mode control of outbreaks of emerging infectious diseases," Bulletin of Mathematical Biology, vol. 74, no. 10, pp. 2403-2422, 2012.

[22] Y. Y. Liu and Y. N. Xiao, "An epidemic model with saturated media/ psychological impact," Applied Mathematics and Mechanics, vol. 34, no. 4, pp. 399-407, 2013.

[23] A. L. Wang and Y. N. Xiao, "A Filippov system describing media effects on the spread of infectious diseases," Nonlinear Analysis: Hybrid Systems, vol. 11, pp. 84-97, 2014.

[24] Y. Xiao, T. Zhao, and S. Tang, "Dynamics of an infectious diseases with media/psychology induced non-smooth incidence," Mathematical Biosciences and Engineering, vol. 10, no. 2, pp. 445-461, 2013.

[25] Y. Xiao, S. Tang, and J. Wu, "Media impact switching surface during an infectious disease outbreak," Scientific Reports, vol. 5, article 7838, 2015.

[26] P. van den Driessche and J. Watmough, "Reproduction numbers and sub-threshold endemic equilibria for compartmental models of disease transmission," Mathematical Biosciences, vol. 180, no. 1-2, pp. 29-48, 2002. 


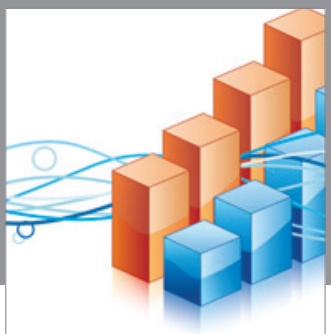

Advances in

Operations Research

vatem alat4

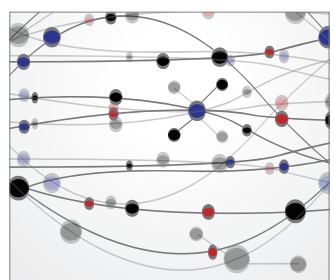

\section{The Scientific} World Journal
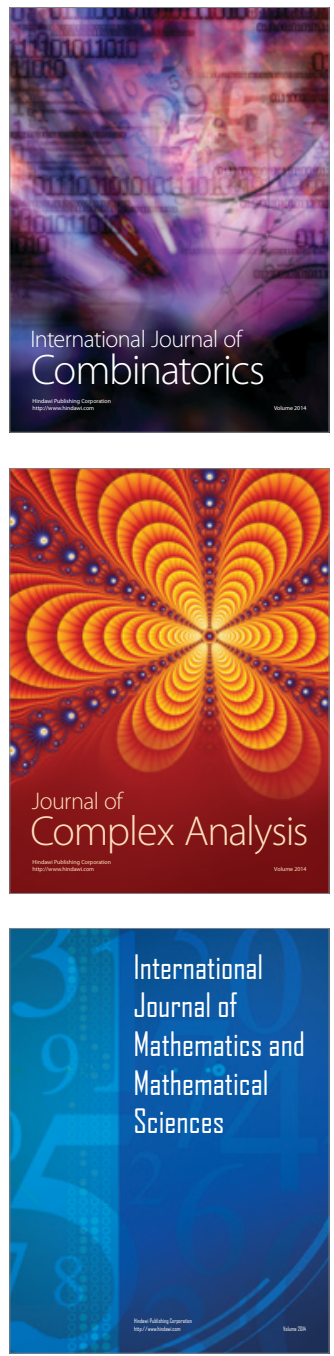
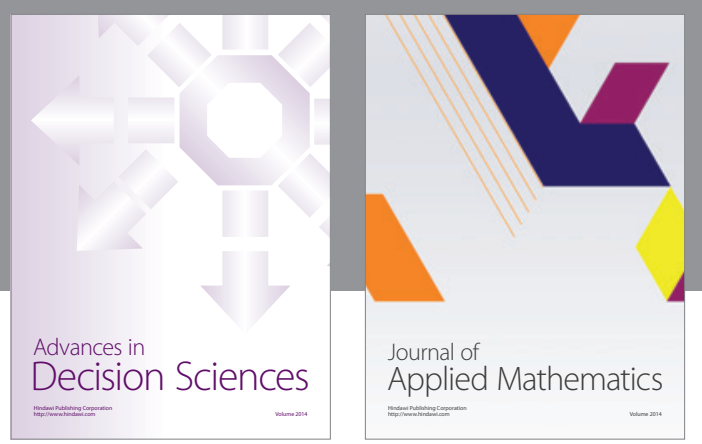

Algebra

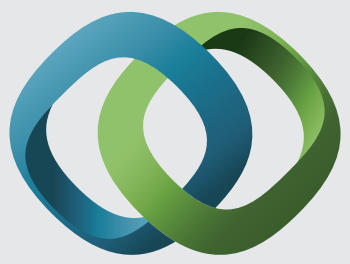

\section{Hindawi}

Submit your manuscripts at

http://www.hindawi.com
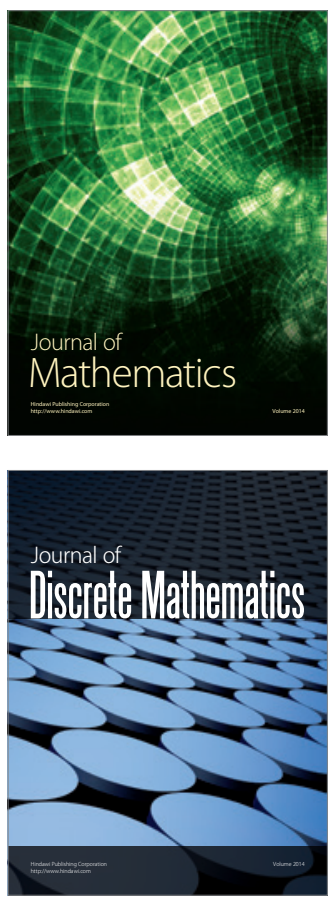

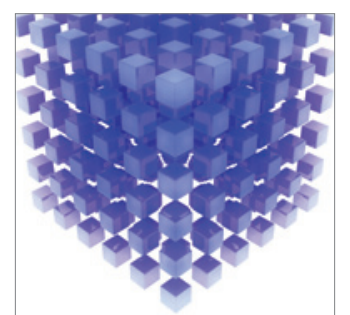

Mathematical Problems in Engineering
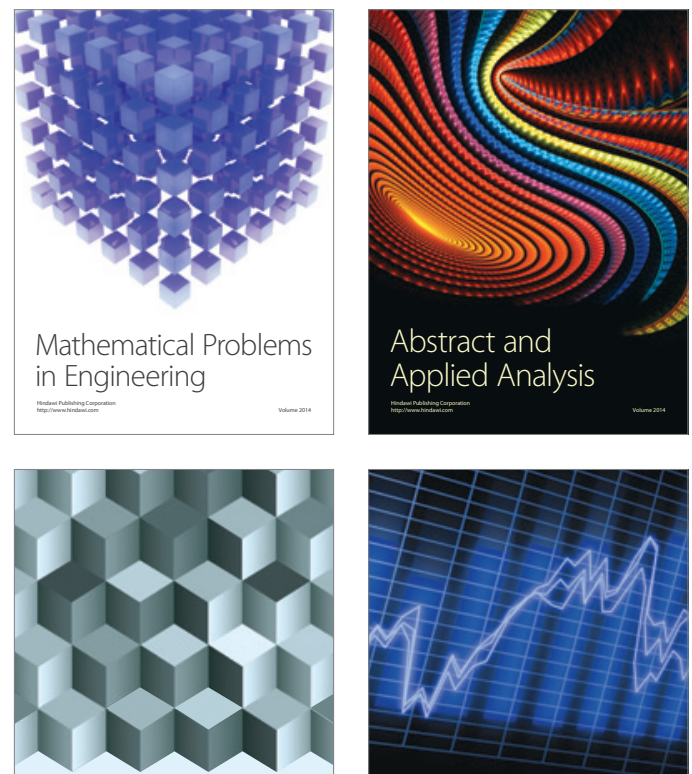

Journal of

Function Spaces

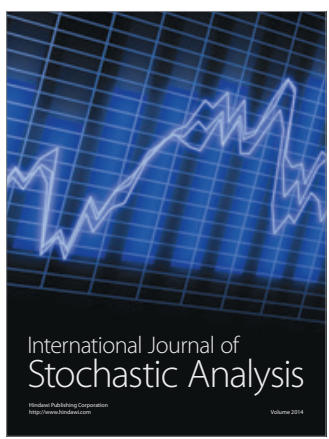

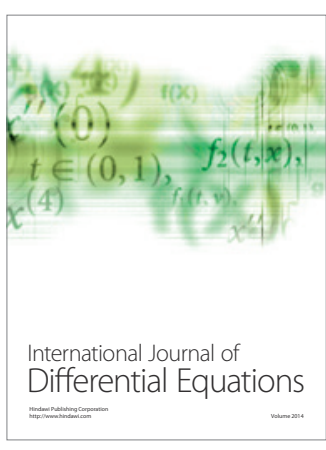
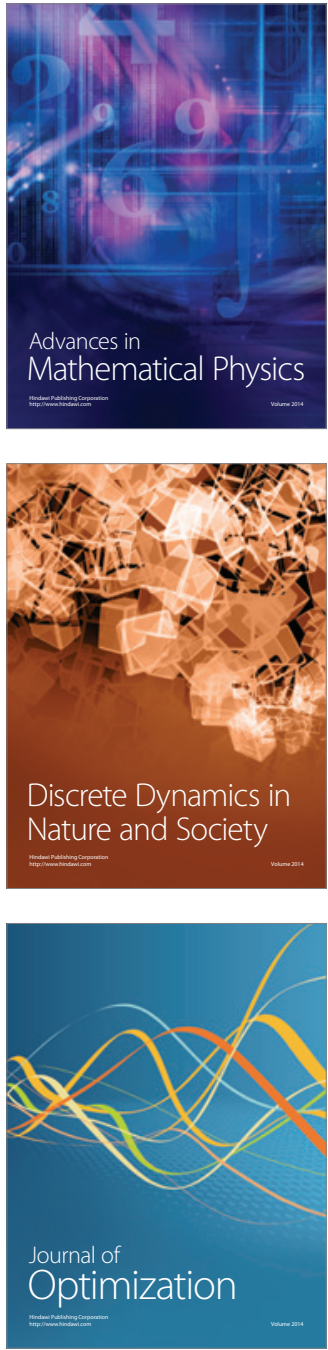